\title{
An investigation of cheapest-to-deliver on Treasury bond futures contracts
}

\author{
Simon Benninga and Zvi Wiener
}

It is commonly believed that the cheapest-to-deliver bond on a Treasury bond futures contract has extremal duration. The authors show that this is not always true. There is an easy rule for cheapest-to-deliver bonds which involves choosing a combination of extremal coupons and maturities. This rule is derived for a flat term structure and its extension to a nonflat term structure is given.

\section{INTRODUCTION}

In this paper we reconsider the problem of the cheapest-to-deliver (CTD) on a Treasury bond (T-bond) futures contract. This problem has received a great deal of attention in the finance literature; interest in it derives from the fact that the Treasury bond futures contract - one of the most widely traded of all financial futures contracts - allows for the delivery of a wide range of Treasury bonds and that the procedure for adjusting the delivery price of these bonds rarely conforms to the differences in market prices.

An extensive literature dealing with the CTD when the term structure is flat has somehow become mired in the misconception that the CTD is characterizable in terms of the duration. We show that this is not always true and that the exceptions to the duration rule contain a large number of economically relevant scenarios. ${ }^{1}$ Another branch of the literature on this topic has examined the problem by using specific term structure models to examine the valuation of the options implicit in the delivery choice. ${ }^{2}$ We prove that the long-term interest rates-most relevant for the CTD decision-must be almost flat in any economically reasonable term structure model, so that the flat term structure case will be a good approximation to a more sophisticated term structure model.

The paper is organized as follows. In Section 2 we set out a simple pricing model for Treasury bond futures. Section 3 shows that the CTD decision in this model-irrespective of the term structure model being used - will always lead to the choice of a CTD having an extremal coupon. In Section 4 we examine the choice of the maturity of the CTD in the context of a flat term structure and

\footnotetext{
${ }^{1}$ We have not succeeded in tracking down the source of this misconception. Jones (1985) - basing his analysis on papers by Kilcollin (1982) and Kane and Marcus (1984) - seems to have been the first to have stated that duration is the determining factor in choosing the CTD. Similar statements are to be found in textbooks (e.g. Edwards and Ma 1992) and in the internal manuals of a number of investment banks.

${ }^{2}$ See, for example, Kane and Marcus (1986), Boyle (1989), Hanweck (1995), Hemler (1990), and Ronn and Bliss (1994).
} 
show that this choice can be characterized into one of four categories. Section 5 compares our characterization with the duration rule common in the literature. We show that in three out of the four cases the duration rule can be different (and in two of these three cases is likely to be different) from the correct CTD rule. Section 6 proves that limiting term structures must be flat, and Section 7 contains some numerical simulations for the Vasicek term structure, a typical nonflat term structure; it can be seen from these simulations that our characterization of the CTD still holds. We summarize our results in Section 8.

\section{A GENERAL MODEL OF THE CTD}

In this section we set out a general model of the CTD for any term structure model. For convenience, we shall use notation which assumes a one-factor term structure model, but extensions to more factors are trivial.

- The discount factor at time $t$ for a $\$ 1$ payment at time $\tau \geqslant t$ when the time- $t$ spot rate is $r$ is denoted by $v(t, \tau, r)$.

- The set of all deliverable T-bonds is assumed to be convex; bonds are assumed to pay continuous coupons. By writing $\{c, M\}$, we denote a T-bond paying a continuous coupon $c$ and having maturity $M$.

- $g(t, T, c, M, r)$ denotes a bond-specific forward contract at time $t$; the contract calls for delivery of a T-bond $\{c, M\}$ at time $T$.

- We ignore the effects of marking to market on the price of the futures contract. This enables us to examine only a forward contract. ${ }^{3}$

- The price today of a T-bond with coupon $c$ and maturity $M$ is given by

$$
\operatorname{price}(c, M)=\int_{t}^{M} c \cdot v(t, \tau, r) \mathrm{d} \tau+v(t, M, r) .
$$

- $\mathrm{CF}(c, M, T)$-the conversion factor for a T-bond with maturity $M$ and coupon $c$ delivered at time $T$ against the forward contract-is calculated using a continuous version of the CBOT formula:

$$
\begin{aligned}
\mathrm{CF}(c, M, T) & =\int_{0}^{M-T} c \cdot \mathrm{e}^{-0.08 \tau} \mathrm{d} \tau+e^{-0.08(M-T)} \\
& =\left.\frac{c \mathrm{e}^{-0.08 \tau}}{-0.08}\right|_{0} ^{M-T}+\mathrm{e}^{-0.08(M-T)} \\
& =\frac{c\left(1-\mathrm{e}^{-0.08(M-T)}\right)}{0.08}+\mathrm{e}^{-0.08(M-T)} .
\end{aligned}
$$

\footnotetext{
3 Although in principle marking to market is a factor in futures versus forward pricing (see e.g. Cox, Ingersoll, and Ross 1981, Jarrow and Oldfield 1981, Richard and Sundaresan 1981), there seems to be general agreement that marking to market is not an important pricing consideration for financial futures contracts. See Sundaresan (1991), Benninga and Protopapadakis (1994), and Hanweck (1995).
} 


\section{Nonoption Forward Contracts}

Suppose that we are offered a nonoption forward contract on a specific bond $\{c, M\}$ at time $t$, and suppose that the forward price of this contract is $g(t, T, c, M, r) .{ }^{4}$ Denote by $f(t, T, c, M, r)$ the profit from buying the specific bond $\{c, M\}$ today and holding it for delivery on the forward contract. It is clear that the forward contract will be priced so that the profit to its participants will be zero:

$$
\begin{aligned}
f(t, T, c, M, r) & \\
& =v(t, T, r) g(t, T, c, M, r) \operatorname{CF}(c, M, T)-\left(\int_{T}^{M} c \cdot v(t, \tau, r) \mathrm{d} \tau+v(t, M, r)\right) \\
& =0 \\
& \Rightarrow g(t, T, c, M, r)=\frac{\int_{T}^{M} c \cdot v(t, \tau, r) \mathrm{d} \tau+v(t, M, r)}{v(t, T, r) \mathrm{CF}(c, M, T)} .
\end{aligned}
$$

For the moment we assume that the set of deliverable bonds is convex and compact; convexity implies that if $\left\{c_{1}, M_{1}\right\}$ is the coupon and maturity on a particular deliverable bond and $\left\{c_{2}, M_{2}\right\}$ is a combination of coupon and maturity on a second bond, then $\left\{\lambda c_{1}+(1-\lambda) c_{2}, \lambda M_{1}+(1-\lambda) M_{2}\right\}$ is also the specification of a deliverable bond. ${ }^{5}$

In the T-bond futures contract, the short chooses the delivery instrument. This means that the forward price $g(t, T, c, M, r)$ which minimizes the above function for all deliverable $\{c, M\}$ will be the market forward price. The next two sections discuss the derivation of this forward price.

\section{THE EXTREMAL COUPON AS A GENERAL SOLUTION FOR THE CTD}

Denote by $g^{*}(t, T)$ the minimum of $g(t, T, c, M, r)$ for all $\{c, M\}$. Suppose this minimum is achieved for $\left\{c^{*}(t, T, r), M^{*}(t, T, r)\right\}$; where no confusion arises, we shall write $\left\{c^{*}, M^{*}\right\}$. Proposition 1 shows that $\left\{c^{*}, M^{*}\right\}$ is always achieved for either the highest or lowest coupon bond, independent of any assumptions on the term structure.

Proposition 1 Given maturity $M, g^{*}(t, T, r)=\min _{\{c, M\}} g(t, T, c, M, r)$ is achieved for extremal $c$.

\footnotetext{
4 The contract $g(t, T, c, M, r)$ is a version of a standard bond repo contract.

${ }^{5}$ The assumptions on convexity and compactness of the set of deliverable bonds is standard in the CTD literature, although it is not always made explicitly. Compactness is a fairly harmless assumption, but the assumption of convexity is not entirely trivial. For example, consider the convex combination of two bonds: if the bonds have the same maturity but different coupons, this convex combination will give a bond with an intermediate coupon; however, the convex combination of two bonds having the same coupon but different maturities is not a bond with an intermediate maturity.
} 
Proof. We want to find $g^{*}(t, T, r)=\min _{\{c, M\}} g(t, T, c, M, r)$. This means taking the derivative of $g(t, T, c, M, r)$ with respect to $c$ and $M$. First, consider the derivative with respect to $c$. As a function of $c, g(t, T, c, M, r)$ is a very simple function of the type

$$
\frac{A c+B}{Q c+D}
$$

The derivative of this function is

$$
\frac{A(Q c+D)-Q(A c+B)}{(Q c+D)^{2}}=\frac{A D-Q B}{(Q c+D)^{2}} .
$$

The denominator of this function is positive, so that the sign depends only on $A D-Q B$, so that it follows that the sign of the derivative with respect to $c$ is either positive or negative; i.e. we will always choose the extremal $c$. Below, we define $A, B, Q, D$ :

$$
\begin{aligned}
& A=\int_{T}^{M} v(t, \tau, r) \mathrm{d} \tau, \quad B=v(t, M, r) \\
& Q=v(t, T, r)\left(\frac{1-\mathrm{e}^{-0.08(M-T)}}{0.08}\right), \quad D=v(t, T, r) \mathrm{e}^{-0.08(M-T)} .
\end{aligned}
$$

Then it follows that

$$
\begin{aligned}
A D & -Q B \\
& =v(t, T, r) \mathrm{e}^{-0.08(M-T)} \int_{T}^{M} v(t, \tau, r) \mathrm{d} \tau-v(t, T, r) v(t, M, r)\left(\frac{1-\mathrm{e}^{-0.08(M-T)}}{0.08}\right) \\
& =v(t, T, r)\left[\mathrm{e}^{-0.08(M-T)} \int_{T}^{M} v(t, \tau, r) \mathrm{d} \tau-v(t, M, r)\left(\frac{1-\mathrm{e}^{-0.08(M-T)}}{0.08}\right)\right]
\end{aligned}
$$

The proposition is now clear, since this expression does not depend on $c$.

\section{CHOOSING THE OPTIMAL MATURITY FOR CTD: THE CASE OF FLAT TERM STRUCTURE}

In Section 3 we showed that-independent of the term structure model - the optimal CTD will have either the highest or the lowest coupon $c$. In this section we consider the optimal maturity of the CTD for the case of a flat term structure. In Section 6 we show (Proposition 4) that all economically reasonable term structures are approximately flat for large maturities. Since the T-bond futures contract is written on long-maturity bonds, we shall claim that the assumption of flat term structure is a good approximation of the results for actual term structures. In Section 7 we examine a typical nonflat term structure 
model and give some numerical results which confirm that the flat term structure is a reasonable approximation for the CTD problem.

In order to set the stage, we first prove the following proposition, which extends the results of Proposition 1 for the case of a flat (or approximately flat) term structure.

Proposition 2 When the long-run term structure is approximately flat (and writing $r_{\mathrm{L}}$ as the long-run interest rate), we can show that $A D-Q B>0$ if and only if $r_{\mathrm{L}}>8 \%$. This means that when $r_{\mathrm{L}}>8 \%$, we will choose the lowest coupon, and when $r_{\mathrm{L}}<8 \%$, we will choose the highest coupon $c$ for delivery.

Proof. Using the notation of Proposition 1, the assumption of flat term structure for long periods can be expressed as

Thus,

$$
A=\int_{T}^{M} \mathrm{e}^{-r_{\mathrm{L}}(\tau-t)} \mathrm{d} \tau=\mathrm{e}^{r_{\mathrm{L}} t} \frac{\mathrm{e}^{-r_{\mathrm{L}} T}-\mathrm{e}^{-r_{\mathrm{L}} M}}{r_{\mathrm{L}}}, \quad B=\mathrm{e}^{-r_{\mathrm{L}}(M-t)} .
$$

$A D-Q B$

$$
\begin{aligned}
& =\mathrm{e}^{-0.08(M-T)} \mathrm{e}^{r_{\mathrm{L}} t} \frac{\mathrm{e}^{-r_{\mathrm{L}} T}-\mathrm{e}^{-r_{\mathrm{L}} M}}{r_{\mathrm{L}}}-\mathrm{e}^{-r_{\mathrm{L}}(M-t)} \frac{1-\mathrm{e}^{-0.08(M-T)}}{0.08} \\
& =\mathrm{e}^{r_{\mathrm{L}} t}\left(\mathrm{e}^{-0.08(M-T)} \mathrm{e}^{-r_{\mathrm{L}} T} \frac{1-\mathrm{e}^{-r_{\mathrm{L}}(M-T)}}{r_{\mathrm{L}}}-\mathrm{e}^{-r_{\mathrm{L}} M} \frac{1-\mathrm{e}^{-0.08(M-T)}}{0.08}\right) \\
& =\mathrm{e}^{r_{\mathrm{L}}(t-T)}\left(\mathrm{e}^{-0.08(M-T)} \frac{1-\mathrm{e}^{-r_{\mathrm{L}}(M-T)}}{r_{\mathrm{L}}}-\mathrm{e}^{-r_{\mathrm{L}}(M-T)} \frac{1-e^{-0.08(M-T)}}{0.08}\right) \\
& =\mathrm{e}^{r_{\mathrm{L}}(t-T)}\left(\frac{\mathrm{e}^{-0.08(M-T)}-\mathrm{e}^{-\left(r_{\mathrm{L}}+0.08\right)(M-T)}}{r_{\mathrm{L}}}-\frac{\mathrm{e}^{-r_{\mathrm{L}}(M-T)}-\mathrm{e}^{-\left(r_{\mathrm{L}}+0.08\right)(M-T)}}{0.08}\right) .
\end{aligned}
$$

The sign of $A D-Q B$ is defined by the sign of the expression in parentheses. This expression can be rewritten using the following notation. Let $a=0.08$, $b=r_{\mathrm{L}}, \tau=M-T$; then we have to define the sign of

$$
\frac{\mathrm{e}^{-a \tau}-\mathrm{e}^{-(a+b) \tau}}{b}-\frac{\mathrm{e}^{-b \tau}-\mathrm{e}^{-(a+b) \tau}}{a}=\mathrm{e}^{-(a+b) \tau}\left(\frac{\mathrm{e}^{b \tau}-1}{b}-\frac{\mathrm{e}^{a \tau}-1}{a}\right),
$$

which is the same as the sign of

$$
\frac{\mathrm{e}^{b \tau}-1}{b}-\frac{\mathrm{e}^{a \tau}-1}{a}
$$

The last expression is zero when $a=b$. To define its sign for other values, we can study the function $\left(\mathrm{e}^{x \tau}-1\right) / x$. This function has the following derivative:

$$
\frac{\mathrm{d}}{\mathrm{d} x} \frac{\mathrm{e}^{x \tau}-1}{x}=\frac{x \tau \mathrm{e}^{x \tau}-\left(\mathrm{e}^{x \tau}-1\right)}{x^{2}} .
$$

Since $x$ is always positive, the sign of the derivative is defined by the numerator, 
which can be written as (using the substitution $y=x \tau$ ) as $y \mathrm{e}^{y}-\mathrm{e}^{y}+1$. It is an easy exercise to show that this function is always positive for $y>0$. This follows since, when $y$ tends to 0 , the value of the expression goes to zero. For positive $y$, its derivative is $\mathrm{e}^{y}+y \mathrm{e}^{y}-\mathrm{e}^{y}=y \mathrm{e}^{y}>0$, which means that the function is positive when $y>0$. This implies that the function $\left(\mathrm{e}^{x \tau}-1\right) / x$ increases when $x$ increases (regardless of the value of $\tau$ ). Thus the expression

$$
\frac{\mathrm{e}^{b \tau}-1}{b}-\frac{\mathrm{e}^{a \tau}-1}{a}
$$

is positive when $b>a$ and negative otherwise, for all values of $\tau$. This means that the expression $A D-B Q$ is always positive when the long-term interest rate $r_{\mathrm{L}}$ is bigger than $8 \%$ and negative otherwise. Note that, when $r_{\mathrm{L}}$ is exactly $8 \%$, then under the assumption of flat long-term interest rates the derivative is zero and $g$ does not depend on $c$.

Proposition 2 shows that, when the term structure is flat, if interest rates are above $8 \%$ it will be optimal to deliver the smallest coupon and vice versa. In Proposition 3, we show the taxonomy of Table 1 for the optimal choice of $\{c, M\}$ in the case of a flat term structure.

Proposition 3 If the term structure is flat, then $g^{*}(t, T, r)=$ $\min _{\{c, M\}} g(t, T, c, M, r)$ is determined by Table 1 .

Proof. When the term structure is flat, the formula for the present value factor becomes $v\left(t_{1}, t_{2}, r\right)=\mathrm{e}^{-r\left(t_{2}-t_{1}\right)}$, and the formula for $g$ becomes

$$
\begin{aligned}
g(t, T, c, M, r) & =\frac{(c / r)\left(\mathrm{e}^{-r(T-t)}-\mathrm{e}^{-r(M-t)}\right)+\mathrm{e}^{-r(M-t)}}{\mathrm{e}^{-r(T-t)}\left[(c / 0.08)\left(1-\mathrm{e}^{-0.08(M-T)}\right)+\mathrm{e}^{-0.08(M-T)}\right]} \\
& =\frac{(c / r)\left(1-\mathrm{e}^{-r(M-T)}\right)+\mathrm{e}^{-r(M-T)}}{(c / 0.08)\left(1-\mathrm{e}^{-0.08(M-T)}\right)+\mathrm{e}^{-0.08(M-T)}} .
\end{aligned}
$$

TABLE 1. Characterization of optimal CTD.

\begin{tabular}{clll}
\hline Case & Characterization & Optimal coupon & Optimal $M$ \\
\hline 1 & $8 \%>r>\max c$ & Largest $c$ & $\begin{array}{l}\text { Smallest } M \text { (there could be another } \\
\text { local minimum but this is unrealistic } \\
\text { since the value of } M \text { is usually larger } \\
\text { than } 50 \text { years) }\end{array}$ \\
& & Largest $c$ & $\begin{array}{l}\text { Smallest } M \\
\text { Largest } M\end{array}$ \\
3 & $\begin{array}{l}r \% 8 \%, \\
\min c>8 \%\end{array}$ & Smallest $c$ & max $M \geqslant M^{*} \geqslant \min M$, with possibility \\
& $r>8 \%>\min c$ & Smallest $c$ & of an interior optimum. \\
\hline
\end{tabular}


To find the cheapest contract, we differentiate $g$ at the optimal coupon $c^{*}$ with respect to $M$ :

$$
\begin{aligned}
& \frac{\partial g\left(t, T, c^{*}, M, r\right)}{\partial M} \\
& \quad=\frac{\mathrm{e}^{M(0.08-r)} 0.08\left(c^{*}-r\right)}{c^{*} \mathrm{e}^{0.08 M}+0.08-c^{*}}+\frac{\mathrm{e}^{M(0.08-r)}\left(0.08-c^{*}\right) 0.08^{2}\left(c^{*} \mathrm{e}^{M r}+r-c^{*}\right)}{\left(c^{*} \mathrm{e}^{0.08 M}+0.08-c^{*}\right)^{2} r} .
\end{aligned}
$$

It is now an easy exercise to find $M^{*}$ where there is a minimum of $g$. Since we are interested in the sign of the partial derivative of $g$ with respect to $M$, we can multiply the expression above by

$$
\frac{c^{*} \mathrm{e}^{0.08 M}+0.08-c^{*}}{0.08 \mathrm{e}^{M(0.08-r)}}>0,
$$

which does not change sign and gives

$$
\frac{\partial g}{\partial M}=\left(c^{*}-r\right)-\left(c^{*}-0.08\right) \frac{0.08\left(c^{*} \mathrm{e}^{M r}+r-c^{*}\right)}{r\left(c^{*} \mathrm{e}^{0.08 M}+0.08-c^{*}\right)} .
$$

We can now easily solve Cases $1-4$. Since the proofs of Cases 1 and 4 are the most difficult and the results are more equivocal, we start with Cases 2 and 3.

Case 2a: $8 \%>\max c=c^{*}>r$. Here $c^{*}-r>0$ and $c^{*}-0.08<0$, so the derivative is positive and the optimal $M$ is the smallest one.

Case 2b: $\max c=c^{*}>8 \%>r$. Here $c^{*}-r>c^{*}-0.08$ and in addition the fraction that multiplies $c^{*}-r$ is less than 1 since $8 \%>r$ (see explanations in Case 1). Thus the derivative is positive and the minimum is achieved at the smallest $M$.

Case 3a: $\min c=c^{*}>r>8 \%$. Then $c^{*}-r<c^{*}-0.08$ and the fraction which multiplies $c-0.08$ is bigger than 1 . Set

$$
f(x)=\frac{c^{*} \mathrm{e}^{M x}+x-c^{*}}{x} .
$$

Then

$$
\frac{\mathrm{d} f}{\mathrm{~d} x}=\frac{1}{x^{2}}\left(x M c \mathrm{e}^{M x}+x-c^{*} \mathrm{e}^{M x}-x+c^{*}\right)=\frac{c^{*}}{x^{2}}\left(x M \mathrm{e}^{M x}-\mathrm{e}^{M x}+1\right) .
$$

We can see that this derivative is proportional to $y \mathrm{e}^{y}-\mathrm{e}^{y}+1$ (here $y=M x$ ), which is always positive. Thus function $f$ always (i.e. for any value of $M$ ) increases when $x$ increases. Note that the fraction that multiplies $c^{*}-0.08$ can be written as $f(r) / f(0.08)$, and since $r>8 \%$ it is bigger than 1 . This implies that the partial of $g$ with respect to $M$ is negative and its minimum is achieved at the largest possible $M$. 
Case 3b: $r>\min c=c^{*}>8 \%$. This case is similar to the Case $3 \mathrm{~b}$, but here even the first expression $c-r$ is negative, which implies that the whole derivative is negative and that the optimal $M$ is the largest possible one.

The proofs for Cases 1 and 4 are somewhat more difficult. We start with Case 4 .

Case 4: $r>8 \%>\min c=c^{*}$. Eliminating like terms in $\partial g / \partial M$, we can write this derivative as proportional to the following expression:

$$
\frac{\partial g}{\partial M}=\left(c^{*}-r\right)-\left(c^{*}-0.08\right) \frac{0.08\left(c^{*} \mathrm{e}^{M r}+r-c^{*}\right)}{r\left(c^{*} \mathrm{e}^{0.08 M}+0.08-c^{*}\right)} .
$$

This expression has two terms, the first of which (for this case) is always negative and the second of which is always positive. Whether the derivative $\partial g / \partial M$ is negative or not thus depends on the relative sizes of these terms. It is easy to see that when $c^{*}$ is close to $8 \%$, the derivative will be zero only for very large $M$. It is only for the case where $8 \%-c^{*}$ is relatively large that $r$ is relatively close to $8 \%$ that the derivative will be negative for reasonable $M$.

More than this is difficult to say without using numerical simulation. The contour plot in Figure 1 shows the locus of combinations of $c$ (on the $x$-axis) and $r$ (on the $y$-axis) for which $\partial g / \partial M=0$ when $M=30$ (note that there are no analytic solutions to $M^{*}$ ). Combinations of $\{c, r\}$ above the line lead to a CTD with $M^{*}<30$. All combinations of $\{c, r\}$ below the line correspond to the situation where the CTD has $M^{*}>30$. Since most reasonable combinations of $\{c, r\}$ are below the line, and since almost always the largest deliverable maturity is less than 30 years, we claim that for this case it is reasonable to state that the optimal deliverable bond will have the longest maturity of available deliverable bonds (this assumes rectangle of deliverability). This concludes the proof of Proposition 3.

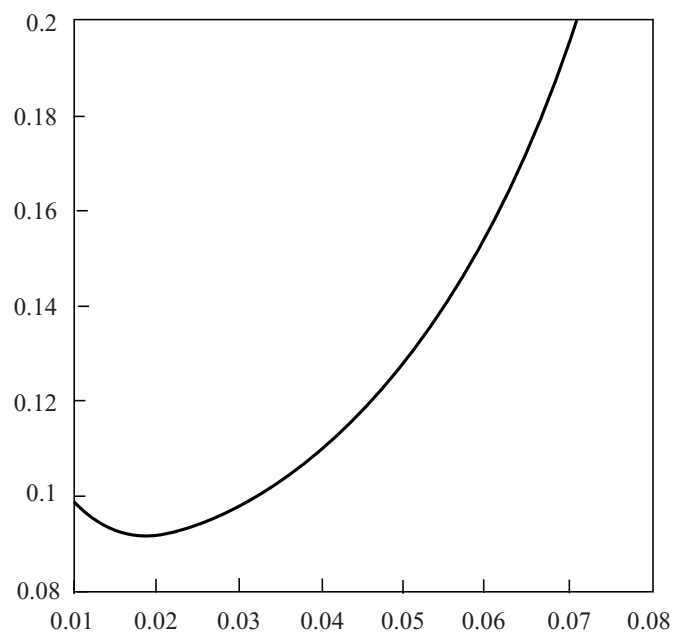

FIGURE 1 
To sum up the results of this and the previous section, we conclude that, independent of the term structure model, the delivery specifications on the Tbond futures contract lead to a CTD with either the lowest or highest coupon. When the term structure is flat, we can completely characterize the optimal maturity of the CTD; we have shown that this will fall into one of four cases. The following section compares our characterization to the commonly cited duration rules in the literature.

\section{THE CTD AND DURATION}

A number of authors (e.g. Jones 1985, Kane and Marcus 1986, p. 236, and Edwards and Ma 1992, p. 333) state that the solution to the above problem (i.e. the CTD bond) when the term structure is flat is the deliverable bond with:

- highest duration if the market interest rate is greater then $8 \%$; and

- lowest duration if the market interest rate is less than $8 \%$.

In this section we discuss this duration-based rule and compare it with our characterization of the optimal delivery problem proved in Propositions $1-3$. We show that the duration-based rule is not true in three out of the four cases in Table 1. For convenience of exposition, we set out, in Table 2, our characterization of the CTD and a comparison with the duration rule.

We now proceed to demonstrate our results.

Case 1: $8 \%>r>\max c$. When $8 \%>r$, it is optimal to choose the largest available coupon for the delivery bond. If this coupon is less than $8 \%$ (this in itself is not a very reasonable case, since it is unlikely that there are no deliverable bonds with coupons less than $8 \%$ ), then we show it is optimal to choose the smallest available $M$. The duration rule for this case is to choose the

TABLE 2. Comparing optimal CTD to duration-based rules.

\begin{tabular}{|c|c|c|c|c|c|}
\hline Case & $\begin{array}{l}\text { Interest } \\
\text { rates }\end{array}$ & $\begin{array}{l}\text { Optimal } \\
\text { coupon }\end{array}$ & Optimal $M$ & $\begin{array}{l}\text { Duration } \\
\text { rule }\end{array}$ & Agreement? \\
\hline 1 & $8 \%>r>\max c$ & Largest $c$ & $\begin{array}{l}\text { In general, } \\
\text { smallest } M\end{array}$ & $\begin{array}{l}\text { Shortest } \\
\text { duration }\end{array}$ & $\begin{array}{l}\text { For most } \\
\text { economically } \\
\text { relevant } \\
\text { cases }\end{array}$ \\
\hline 2 & $\begin{array}{l}8 \%>r \\
\max c>r\end{array}$ & Largest $c$ & Smallest $M$ & $\begin{array}{l}\text { Shortest } \\
\text { duration }\end{array}$ & Always \\
\hline 3 & $\begin{array}{l}r>8 \% \\
\min c>8 \%\end{array}$ & Smallest $c$ & Largest $M$ & $\begin{array}{l}\text { Longest } \\
\text { duration }\end{array}$ & Not always \\
\hline 4 & $r>8 \%>\min c$ & Smallest $c$ & $\begin{array}{l}\max M \geqslant \\
M^{*} \geqslant \min M, \\
\text { with possibility } \\
\text { of an interior } \\
\text { optimum. }\end{array}$ & $\begin{array}{l}\text { Longest } \\
\text { duration }\end{array}$ & Not always \\
\hline
\end{tabular}




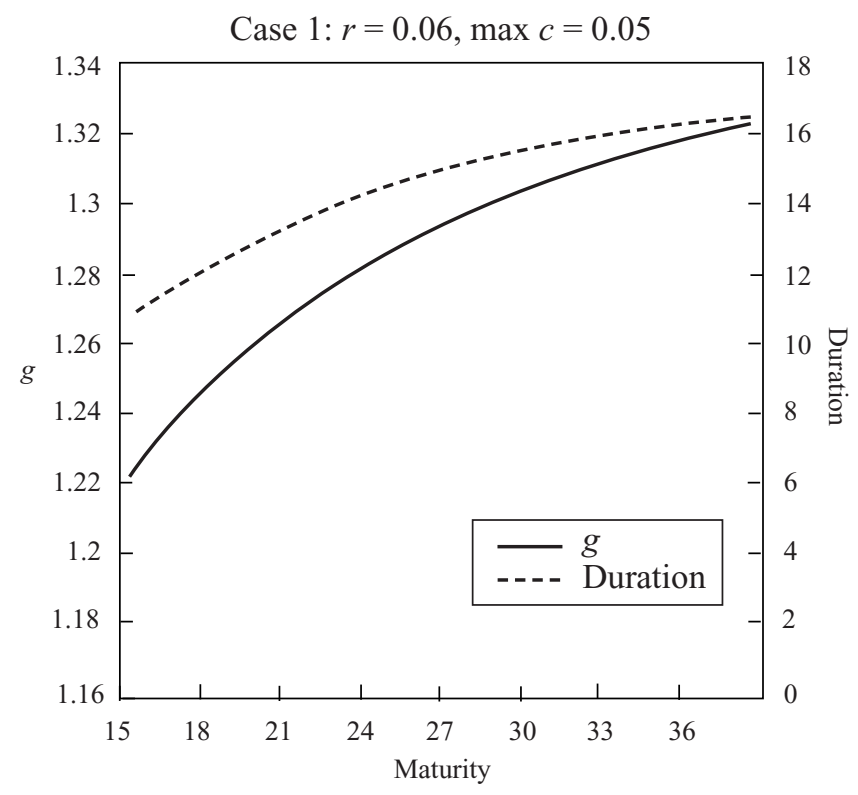

FIGURE 2

smallest duration bond in the deliverable set; for most cases of this type, the duration rule works. Figure 2 shows the case where $r=6 \%$ and $\max c=$ $c^{*}=5 \%$.

We stress that, in this case, the duration rule does not always work. It is easy to construct an example where $8 \%>r>\max c$ for which the duration has an internal maximum, and hence two local minima. However, in order for this internal maximum to be at bond maturities less than 30 years, $r$ must be greater than $8 \%$. Thus the intuitive rule is correct for this case, provided that there are no deliverable T-bonds with maturities longer than 30 years. If very-long-term deliverable bonds exist, it is possible that the smallest $g$ and the lowest duration no longer coincide.

Case 2: $8 \%>r, \max c>r$. When $8 \%>r$, it is optimal to choose the largest available coupon for the delivery bond. If this coupon is greater than $8 \%$, then we show it is optimal to choose the smallest available $M$. For this case, duration increases with increasing bond maturity (see e.g. Bierwag, Kaufman, and Toevs 1983) and it is therefore optimal to choose the bond with the lowest duration. Figure 3 shows the case where $r=6 \%$ and $\max c=c^{*}=11 \%$.

Case 3: $r>8 \%, \min c>8 \%$. When $r>8 \%$, it is optimal to choose the smallest available coupon for the delivery bond. The standard claim in the literature is that it is - in this case-also optimal to choose the highest duration bond. Figure 4 (in which $r=18 \%$ and $\min c=c^{*}=10 \%$ ) shows a counterexample for which this claim does not hold. 


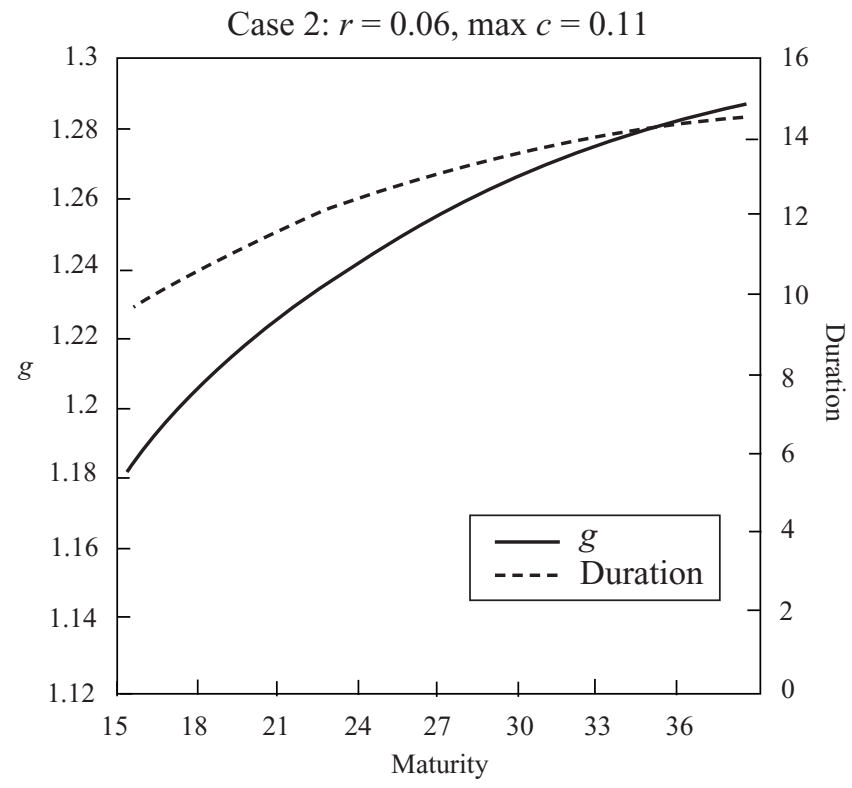

FIGURE 3

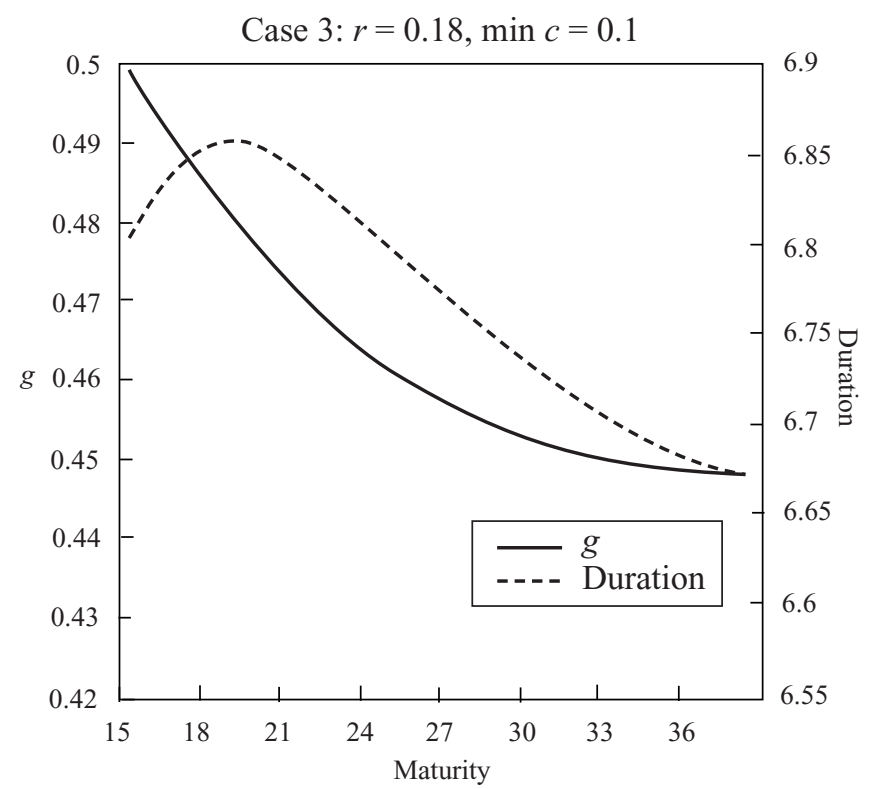

FIGURE 4 


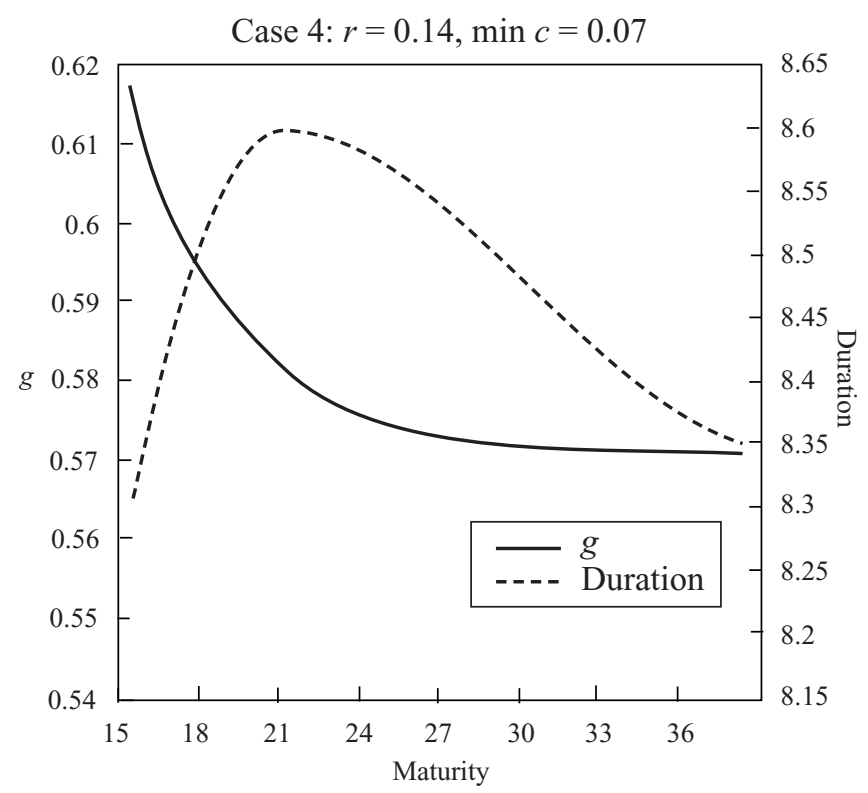

FIGURE 5

The intuition behind this result is that, although a discount bond's duration ultimately declines, it can initially rise with increasing maturity. Thus the duration can have an internal maximum, whereas for this case-as proved in Proposition 3-the function $g$ has a minimum for the largest deliverable maturity.

Case 4: $r>8 \%>\min c$. When $r>8 \%$, it is optimal to choose the smallest available coupon for the delivery bond. If this coupon is smaller than $8 \%$, then it is not optimal to choose the highest duration. As we show in Figure 5, it is easy to construct examples in which the value of $M$ for which the duration is at a maximum is different from the value of $M$ for which $g$ has a minimum (it is this latter value which determines the optimal deliverable bond). In Figure 5, $r=14 \%$ and $\min c=c^{*}=7 \%{ }^{6}$

In summary, for the flat term structure case, whenever $r>8 \%$, the duration rule (which, for this case, would have us choose as CTD a bond with maximal duration) is not true. The duration rule does not necessarily hold for the case where $r<8 \%$ and the optimal deliverable coupon $c^{*}<r$.

\section{THE FLAT TERM STRUCTURE AS A LIMITING CASE}

The analysis of the choice of the optimal maturity for the CTD has concentrated on the case of a flat term structure. In this section we show that the flat term

\footnotetext{
${ }^{6}$ For graphical clarity, we have chosen quite a large $r$, but other examples with smaller values of $r$ can also be constructed.
} 
structure is a reasonable limiting case for the T-bond futures contract. We do this by showing that-under plausible economic circumstances-the term structure is always ultimately flat. Since T-bond futures have as underlying asset a long-term bond, it follows that the logic of the flat term structure case carries over to more general term structure models. In Section 7 we illustrate our contention by showing some simulations for the case of the Vasicek term structure.

Consider an economy with a single representative consumer with a timeadditive utility function. This means that the consumer's expected utility from the lifetime consumption $\tilde{c}=\left\{c_{0}, \tilde{c}_{1}, \tilde{c}_{2}, \ldots\right\}$ is given by

$$
U\left(c_{0}\right)+\sum_{t} \beta^{t} E U\left(\tilde{c}_{t}\right)
$$

where $\beta<1$ is the representative consumer's rate of time preference. ${ }^{7}$ The discount factor for time $t$ is given by

$$
\mathrm{e}^{-r_{t} t}=\beta^{t} \frac{E U^{\prime}\left(\tilde{c}_{t}\right)}{U^{\prime}\left(c_{0}\right)} .
$$

Proposition 4 In a representative consumer model with time-separable concave utility, the term structure ultimately becomes flat provided at least one of the two following conditions holds:

- $\tilde{c}_{t}$ is uniformly bounded away from zero for all $t$;

- $U^{\prime}(0)<\infty$.

Proof. Taking the logarithm of both sides of the above expression and doing some obvious manipulation gives

$$
-r_{t} t=t \ln \beta+\ln \left(\frac{E U^{\prime}\left(\tilde{c}_{t}\right)}{U^{\prime}\left(c_{0}\right)}\right) \Longrightarrow r_{t}=-\ln \beta-\frac{1}{t} \ln \left(\frac{E U^{\prime}\left(\tilde{c}_{t}\right)}{U^{\prime}\left(c_{0}\right)}\right)
$$

Under the assumptions of the proposition, the term $\ln \left(E U^{\prime}\left(\tilde{c}_{t}\right) / U^{\prime}\left(c_{0}\right)\right)$ is bounded since $U^{\prime}$ is a decreasing positive function bounded from above, and hence the limiting interest rate $r_{t}$ converges to $-\ln \beta$ as $t \rightarrow \infty$.

The conditions of Proposition 4 are very general, and the results can be considered as limiting term structure results for economically reasonable general-equilibrium term structure models. ${ }^{8}$

\footnotetext{
${ }^{7}$ Models like this abound in the general equilibrium asset pricing and term structure literature. Examples are papers by Lucas (1978), Benninga and Protopapadakis (1983), and Cox, Ingersoll, and Ross (1985).

${ }^{8}$ Most of the term structure models in the finance literature are not derived in general equilibrium; nevertheless, all converge to flat term structures. The Cox-Ingersoll-Ross model (Cox, Ingersoll, and Ross 1985), which is a term structure model in general equilibrium, uses a representative consumer model of the type described above. The model described in this section is considered in some detail in Wiener, Benninga, and Protopapadakis (1994).
} 


\section{NONFLAT TERM STRUCTURES: SOME NUMERICAL SIMULATIONS WITH THE VASICEK MODEL}

In this section we give some numerical examples of our results for the Vasicek term structure model. The object of this exercise is to show that when the term structure is not flat (and the Vasicek model gives very nonlinear term structures), Table 1 is a very good approximation to the optimal delivery problem. The Vasicek (1978) term structure model assumes that the spot rate $r$ follows an Ornstein-Uhlenbeck process:

$$
\mathrm{d} r=\alpha(\gamma-r) \mathrm{d} t+\rho \mathrm{d} z
$$

where $\gamma$ is the long-term mean spot interest rate and $\rho$ is the instantaneous standard deviation. We denote by $v(t, s, r)$ the Vasicek present value at time $t$ of $\$ 1$ paid at $s$ when the current spot interest rate is $r$. The Vasicek model solves for the present value factors $v(t, s, r)$ at time $t$ for time $s>t$ :

$$
v(t, s, r)=\exp \left(\frac{1}{\alpha}\left(1-\mathrm{e}^{-\alpha(s-t)}\right)[R(\infty)-r]-(s-t) R(\infty)-\frac{\rho^{2}}{4 \alpha^{3}}\left(1-\mathrm{e}^{-\alpha(s-t)}\right)^{2}\right),
$$

where

$$
t \leqslant s
$$

$$
R(\infty)=\gamma+\frac{\rho q}{\alpha}-\frac{\rho^{2}}{2 \alpha^{2}}
$$

Since $R(\infty)$ is the long-run interest rate, and since this interest rate will essentially determine the question of the CTD, we prefer to write the model using $R(\infty)$ as an inputted parameter:

$$
\begin{aligned}
& v(t, s, r, R(\infty)) \\
& \quad=\exp \left(\frac{1}{\alpha}\left(1-\mathrm{e}^{-\alpha(s-t)}\right)[R(\infty)-r]-(s-t) R(\infty)-\frac{\rho^{2}}{4 \alpha^{3}}\left(1-\mathrm{e}^{-\alpha(s-t)}\right)^{2}\right),
\end{aligned}
$$$$
t \leqslant s .
$$

The Vasicek model converges to $R(\infty)$, as shown in Figure 6, which gives the pure-discount interest rate as a function of the time to maturity when the current spot rate $r=9 \%$ and when $R(\infty)=7.5 \% .{ }^{9}$ Although the Vasicek term structure is not derived from an equilibrium model with consumption, it has the property-proved necessary in Section 5-that the long-run interest rates are essentially flat. This means that $R(\infty)$ is in most cases the determinant of the CTD. ${ }^{10}$

\footnotetext{
${ }^{9}$ All our simulations assume that $\alpha=1$.

${ }^{10}$ In some cases it is useful to take a weighted average of the spot rate $r$ and $R(\infty)$.
} 


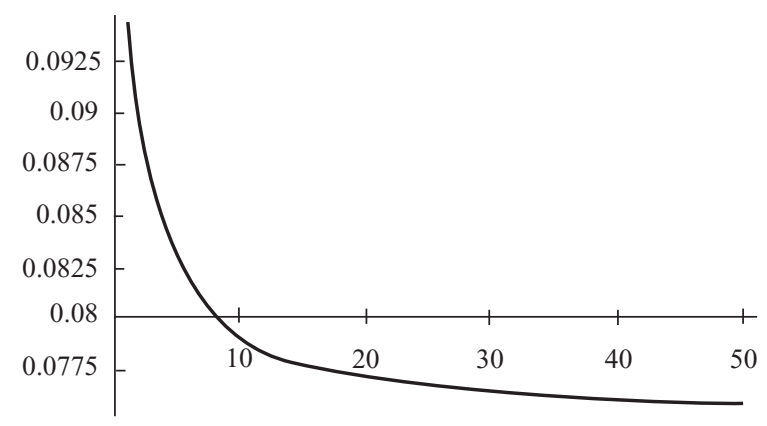

FIGURE 6

When we generate numerical output for the Vasicek model with $R(\infty)$ as the determinant of the CTD, the graphs are very similar to those obtained with the flat term structure. Figure 7, for example, shows a graph of $g$ as a function of $c$ and $M$ for Cases 3 and 4 (i.e. $R(\infty)>8 \%$ ).

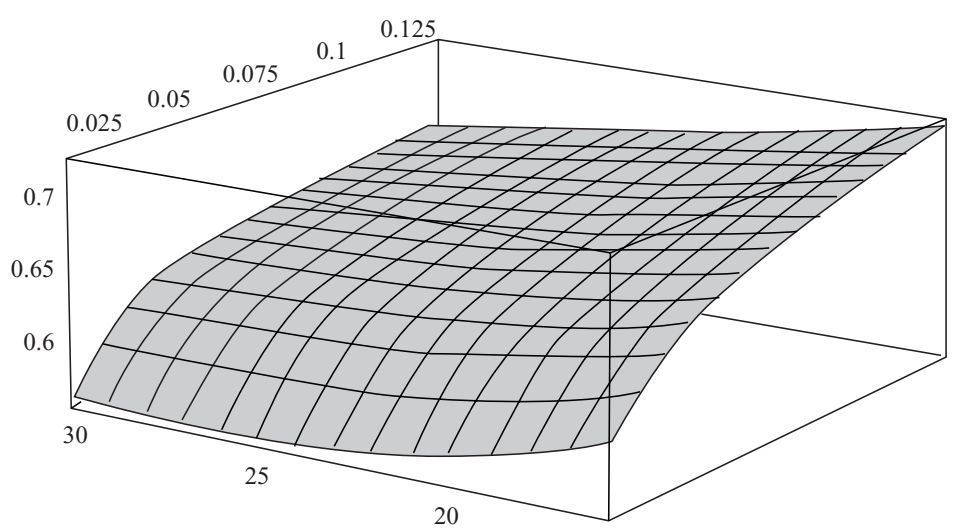

FIGURE 7

As in the case of the flat term structure, the CTD is qualitatively the same (i.e. when $\min c>8 \%$, the CTD has the largest maturity and when $\min c<8 \%$, there is an interior solution for the optimal maturity). Other cases are similar.

\section{CONCLUSIONS}

In this paper we examine the problem of the optimal delivery instrument on the Treasury bond futures contract. Our conclusions may be summarized as follows.

1. For a given bond maturity, the cheapest-to-deliver (CTD) bond always has either the highest (if the market interest rate is less than $8 \%$ ) or lowest coupon of all deliverable bonds. 
2. When the term structure is flat, the maturity of the CTD is the shortest maturity of all deliverable bonds if the market interest rate is less than $8 \%$. If the market interest rate is greater than $8 \%$, the maturity of the CTD is the largest deliverable maturity if the optimal coupon is greater than $8 \%$; if the optimal coupon is less than $8 \%$, then there is the possibility of an optimal deliverable maturity which is neither the largest nor the smallest deliverable maturity (an interior optimum). These results will also hold (approximately) for a nearly flat term structure.

3. In contradistinction to the prevailing (and published) belief, the CTD is - in many cases - not a bond with extremal duration.

4. While Conclusion 2 above is derived for the case of a flat term structure, we have shown that it holds as the only economically plausible limiting case for all term structure models.

\section{Acknowledgements}

We have benefited from the helpful comments of an anonymous referee. We acknowledge funding from the Israeli Academy of Sciences and from the Krueger Center for Finance and Banking of the School of Business, Hebrew University, Jerusalem.

\section{REFERENCES}

Benninga, S., and Protopapadakis, A. (1983). Real and nominal interest rates under uncertainty: The Fisher theorem and the term structure. Journal of Political Economy, 91 (October), 856-867.

Benninga, S., and Protopapadakis, A. (1994). Forward and futures prices with Markovian interest rate processes. Journal of Business, 67, 401-421.

Bierwag, G. O., Kaufman, G. G., and Toevs, A. (1983). Duration: Its development and use in bond portfolio management. Financial Analysts Journal, 39 (July-August), $15-35$.

Boyle, P. (1989). The quality option and the timing option in futures contracts. Journal of Finance, 44, 101-113.

Cox, J. C., Ingersoll, J. E., and Ross, S. A. (1981). The relation between forward and futures prices. Journal of Financial Economics, 9, 321-346.

Cox, J. C., Ingersoll, J. E., and Ross, S. A. (1985). A theory of the term structure of interest rates. Econometrica, 53, 363-384.

Edward, F. R., and Ma, C. W. (1992). Futures \& Options. McGraw-Hill.

Hanweck Jr., G. A. (1995). An asset pricing formula for interest-rate derivative assets with application to Eurodollar futures and option. Working paper. 
Hegde, S. (1988). An empirical analysis of implicit delivery options in the Treasury bond futures contract. Journal of Banking and Finance, 12, 469-492.

Hemler, M. L. (1990). The quality delivery option in Treasury bond futures contracts. Journal of Finance, 45 (December), 1565-1586.

Hull, J. (1991). Introduction to Futures and Options Markets. Prentice-Hall.

Jarrow, R., and Oldfield, G. (1981). Forward contracts and futures contracts. Journal of Financial Economics, 9, 373-382.

Jones, R. A. (1985). Conversion factor risk in Treasury bond futures: Comment. Journal of Futures Markets 5(1), 115-119.

Kane, A., and Marcus, A. J. (1984). Conversion factor risk and hedging in the Treasurybond futures market. Journal of Futures Markets, 4, 55-64.

Kilcollin, T. E. (1982). Difference systems in financial futures markets. Journal of Finance, 37 (December), 1183-1197.

Lucas Jr., R. E. (1978). Asset prices in an exchange economy. Econometrica, 46, 1429-1445.

Richard, S., and Sundaresan, M. (1981). A continuous time equilibrium model of forward prices and futures prices in a multigood economy. Journal of Financial Economics, 9, 321-371.

Ronn, E. I., and Bliss Jr., R. R. (1994). A nonstationary trinomial model for the valuation of options on Treasury bond futures contracts. Journal of Futures Markets, 14, 597-617.

Sundaresan, M. S. (1991). Futures prices on yields, forward prices, and implied forward prices from term structure. Journal of Financial and Quantitative Analysis, 26(3), 409-424.

Vasicek, O. (1977). An equilibrium characterization of the term structure. Journal of Financial Economics, 5, 177-188.

Wiener, Z., Benninga, S., and Protopapadakis, A. (1994). Limiting differences between forward and futures prices in a Lucas consumption model. Working paper, The Rodney L. White Center for Financial Research, The Wharton School, University of Pennsylvania.

\author{
S. Benninga \\ Tel-Aviv University \\ Z. Wiener
}

Hebrew University, Jerusalem 
